N. Van Thu

Nagoya Math. J.

Vol. 133 (1994), 155-175

\title{
GENERALIZED INDEPENDENT INCREMENTS PROCESSES $^{(*)}$
}

\author{
NGUYEN VAN THU
}

\section{Dedicated to Professor K. Urbanik on his 60 th birthday}

We study a class of Markov processes which arise in the theory of generalized convolutions and stand for a generalization of processes with independent increments.

\section{Notation and preliminaries}

Let $P$ be the set of all probability measures (p.m.'s) on the positive half-line $R_{+}=[0, \infty)$ with the weak convergence $\stackrel{w}{\rightarrow}$. We write $\delta_{x}$ for the unit mass at point $x$ and write $T_{x}$ for the map given by

$$
T_{x} \mu(B)=\mu\left(x^{-1} B\right)
$$

for $x>0, \mu \in P$ and $B \in \mathfrak{B}$, the $\sigma$-field of Borel subsets of $R_{+}$. We define $T_{0} \mu=\delta_{0}$. We denote by $Q$ the class of all sub-probability measures (sub-p.m.'s) on $R_{+}$. Let $C_{b}$ be the Banach space of all real bounded continuous functions on $R_{+}$ with supremum norm $\|\cdot\|$ and $C_{0}$ its subspace consisting of functions vanishing at infinity.

A commutative and associative $P$-valued binary operation on $P$ with $\delta_{0}$ as the unit element is called a generalized convolution, if it is continuous in each variable separately and distributive with respect to convex combinations and maps $T_{x}$, and if it satisfies the following law of large numbers:

Received January 8, 1990.

${ }^{*}$ )The research was carried out during the author's stay at Technische Universität Berlin (1988) and at Nagoya Institute of Technology (1989) under grants from Alexander von Humboldt-Stiftung and the Japan Society for the Promotion of Science.

The paper was supported in part by the National Basic Research Program in Natural Science, Vietnam. 
(LLN) There exists a sequence of positive numbers $c_{n}$ such that the sequence $T_{c_{n}} \delta_{1}^{\circ n}$ is convergent to a limit other than $\delta_{0}$.

Here $P^{\circ n}$ denotes the $n$th power of $P$ under the operation.

The pair $\left(P,{ }^{\circ}\right)$ is called a generalized convolution algebra, which was introduced by K. Urbanik in [6] and studied by many researchers (cf. [2], [10], [11], [12], [17-22], [23]).

We assume throughout the paper that the algebra $\left(P,{ }^{\circ}\right)$ is regular, i.e. it admits a characteristic function $\hat{\mu} \in C_{b}$ defined by the following properties: the correspendence $\mu \leftrightarrow \hat{\mu}$ is one-to-one, $\hat{\mu}$ is distributive with respect to convex combinations, $\widehat{\mu_{0} \nu}=\hat{\mu} \hat{\nu}, \widehat{T_{x} \mu}(t)=\hat{\mu}(x t)$, and the uniform convergence of $\hat{\mu}_{n}$ to $\hat{\mu}$ on every finite interval is equivalent to $\mu_{n} \stackrel{w}{\rightarrow} \mu$. The characteristic function $\hat{\mu}$ is represented as

$$
\hat{\mu}(t)=\int \Omega(t x) \mu(d x) .
$$

Here and in the sequel the symbol $\int$ denotes the integral over $[0, \infty)$. The function $\Omega$ is called a kernel of the characteristic function. The system of characteristic functions is unique in the following sense: If there are two systems of characteristic functions with kernels $\Omega_{1}$ and $\Omega_{2}$, respectively, then

$$
\Omega_{1}(t)=\Omega_{2}(c t) \quad(t \geq 0)
$$

for some $c>0$ (cf. Urbanik [18], Theorem 2.1). Henceforth we fix a system of characteristic functions.

The limiting measure in (LLN), denoted by $\sigma_{\varkappa}$, is called the characteristic measure of the algebra in question and (with $c_{n}$ replaced by their constant multiples if necessary) has the following characteristic function:

$$
\hat{\sigma}_{\kappa}(t)=\exp \left(-t^{x}\right)
$$

where $t \geq 0$ and $\kappa$ is a positive constant called the characteristic exponent of the generalized convolution $\circ$. The concepts of infinite divisibility and selfdecomposability are introduced in the algebra $\left(P,{ }^{\circ}\right)$.

In a natural way the operation $\circ$ as well as the characteristic function can be extended to the set $Q$. Moreover, one can also extend the generalized convolution 。 and the map $T_{x}(x>0)$ to the set $\vec{P}$ of all p.m.'s defined on the compactified half-line $\bar{R}_{+}=[0, \infty]$. Namely,

$$
\left(a \mu^{\prime}+(1-a) \delta_{\infty}\right) \circ\left(b \nu^{\prime}+(1-b) \delta_{\infty}\right)=a b\left(\mu^{\prime} \circ \nu^{\prime}\right)+(1-a b) \delta_{\infty},
$$




$$
T_{c}\left(a \mu^{\prime}+(1-a) \delta_{\infty}\right)=a T_{c} \mu^{\prime}+(1-a) \delta_{\infty}
$$

for $0 \leq a \leq 1,0 \leq b \leq 1,0<c<\infty$ and $\mu^{\prime}, \nu^{\prime} \in P$. The pair $\left(\bar{P},{ }^{\circ}\right)$ is called the extended generalized convolution algebra (cf. Urbanik [21]). The concepts of infinitely divisible measures and self-decomposable measures can be defined in terms of the operation $\circ$ also in the extended algebra $\left(\bar{P},{ }^{\circ}\right)$. Consider $\mu \in \bar{P}$ with $\mu=a \mu^{\prime}+(1-a) \delta_{\infty}$ where $\mu^{\prime} \in P$ and $0<a \leq 1$. Then $\mu$ is infinitely divisible in $\left(\bar{P},{ }^{\circ}\right)$ if and only if $\mu^{\prime}$ is infinitely divisible in $\left(P,{ }^{\circ}\right)$. Similarly, $\mu$ is self-decomposable in $\left(\bar{P},{ }^{\circ}\right)$ if and only if $\mu^{\prime}$ is self-decomposable $\left(P,{ }^{\circ}\right)$.

Now we quote some examples of regular generalized convolutions which will be needed in the subsequent discussion. The examples will be given in terms of the kernel $\Omega$ and the characteristic measure $\sigma_{\varkappa}$ or its density $g_{\varkappa}$. Except Example 4 , which was essentially considered by S. Cambanis, R. Keener and G. Simons in [4], the examples can be found in Urbanik's and Kingman's standard papers [16, 17, 18] [10]. The symmetric unimodal convolution in Example 3 and relation (1.3) are given by N. V. Thu.

EXAmple 1. $\alpha$-convolutions $\quad *_{\alpha}(0<\alpha<\infty): \Omega(t) \quad \exp \left(-t^{\alpha}\right), \kappa=\alpha$, $\sigma_{\varkappa}=\delta_{1}$. For $\alpha=1$ we get the ordinary convolution i.e. $*_{1}=*$

EXAmple 2. Symmetric convolution $*_{1,1}: \Omega(t)=\cos t, \kappa=2$,

$$
g_{x}(x)=\frac{1}{\sqrt{\pi}} \exp \left(-4^{-1} x^{2}\right) .
$$

EXAmple 3. Kingman convolutions $\left.*_{1, \beta}(\beta=2(s+1))>1\right)$ : We have $\kappa=2$,

$$
\Omega(t)=\Lambda_{s}(t)=\Gamma(s+1) J_{s}(t) /\left(\frac{1}{2} t\right)^{s},
$$

where $J_{s}$ is the Bessel function and

$$
g_{\kappa}(x)=2^{-2 s-1} x^{2 s+1} \exp \left(-4^{-1} x^{2}\right) / \Gamma(s+1) .
$$

The limiting case $s=-\frac{1}{2}$ reduces to the symmetric convolution. Moreover, as observed by Bingham [2], every Kingman convolution is subordinate to the symmetric convolution:

The case $\beta=3, s=\frac{1}{2}$ reduces to the following symmetric unimodal convolu- 
tion.

Let $W$ denote the uniform distribution on $[-1,1]$. For two independent random variables $X$ and $Y$ with distributions $F$ and $G$ we denote by $F G$ the distribution of the product $X Y$. By Khintchine-Shepp representation (cf. e.g. [6], Theorem 1.5 , p. 10), every symmetric unimodal distribution $\mu$ on the real line can be uniquely represented by $\mu=F W$ with $F \in P$. Furthermore, by a routine computation we have the following equation:

$$
F W * G W=\left(F *_{1,3} G\right) W \quad(F, G \in P),
$$

which is a more specific form of the well-known theorem of Wintner (cf. [24]) asserting that the convolution of two symmetric unimodal distributions on $R$ is unimodal.

EXAMPle 4. $n$-symmetric convolutions $\square_{n}(n=2,3, \ldots)$ : These convolutions appear in the contex of $\alpha$-symmetric distributions (cf. [4]). We have $\kappa=1$,

$$
\Omega(t)=E \Lambda_{s}(t / \sqrt{D}),
$$

with $n=2(s+1)$ and $D$ being a random variable with Beta $\left(\frac{1}{2}, \frac{n-1}{2}\right)$ distribution, and

$$
g_{\varkappa}(x)=\frac{2 \Gamma\left(s+\frac{3}{2}\right)\left(2 x^{2 s+1}\right)}{\sqrt{\pi} \Gamma(s+1)\left(1+x^{2}\right)^{2 s+1}} .
$$

The paper is organized as follows: in $\S 2$ we introduce generalized independent increments processes (०-i.i. processes) and -Lévy processes. We prove that - -Lévy processes are strong Markov Feller processes. In $\S 3$ the infinitesimal genarators associated with -Lévy processes are studied. Generalized Bernstein functions are discussed in $\S 4$. Finally, in $\S 5$ we obtain analogues of some of Sato's and Lamperti's results on self-similar processes (cf. [13], [15]).

\section{Generalized independent increments processes}

Suppose that $\mu_{s, t}(0 \leq s<t)$ is a family of p.m.'s on $\bar{R}_{+}$such that the following eguation is satisfied:

$$
\mu_{s, t}{ }^{\circ} \mu_{t, u}=\mu_{s, u} \quad(0 \leq s<t<u) .
$$

For every $x$ in $\bar{R}_{+}$and $B \in \overline{\mathfrak{B}}, \overline{\mathfrak{V}}$ being the Borel $\sigma$-field of $\bar{R}_{+}$, we put 


$$
P_{s, t}(x, B)=\delta_{x} \circ \mu_{s, t}(B) .
$$

This definition and (2.1) imply the Chapman-Kolmogorov equation

$$
\int P_{s, t}(x, d y) P_{t, u}(y, B)=P_{s, u}(x, B) \quad(0 \leq s<t<u),
$$

which can be proved by characteristic functions. Hence, there exists a $\bar{R}_{+}$-valued Markov process $\left\{X_{t}\right\}$ with transition probability $P_{s, t}$ given by (2.2), that is

$$
P\left(X_{t} \in B \mid X_{u}, u \leq s\right)=P_{s, t}\left(X_{s}, B\right) .
$$

The probability measure under the initial condition $X_{0}=x$ is denoted by $P^{x}$. As usual the expectation with respect to $P^{x}$ is denoted by $E^{x}$.

If $\circ$ is the ordinary convolution then $\left\{X_{t}\right\}$ is a process with independent increments. Therefore, in general case, $\left\{X_{t}\right\}$ will be referred to as a generalized independent increments process, or more precisely, --independent increments process (०-i.i. process).

We say that a family of p.m.'s $\left\{\mu_{t}\right\}$ in $\bar{P}$ is a generalized convolution semigroup (shortly, $\circ-$ semigroup), if the following conditions are satisfied:

$$
\begin{gathered}
\mu_{t} \circ \mu_{s}=\mu_{t+s} \quad(t, s \geq 0) \\
\mu_{t} \stackrel{w}{\rightarrow} \delta_{0} \quad \text { as } \quad t \rightarrow 0 .
\end{gathered}
$$

It follows that $\mu_{0}=\delta_{0}$.

It is easily seen that if $\left\{\mu_{t}\right\}$ is an $\circ$-semigroup then the family $\left\{\mu_{s, t}\right\}$ given by

$$
\mu_{s, t}=\mu_{t-s} \quad(0 \leq s<t)
$$

satisfies (2.1) and induces a time-homogenous o-i.i. process $\left\{X_{t}\right\}$ which will be called in the sequel an -Lévy process.

For an extended generalized convolution algebra $\left(\bar{P},{ }^{\circ}\right)$ define generalized translation operators by

$$
\left(\tau^{a} f\right)(x)=\int^{-} f(u) \delta_{a} \delta_{x}(d u)
$$

where $a, x \in \bar{R}_{+}$and $f$ is a continuous function on $\bar{R}_{+}$. Here and in the sequel $\int^{-}$denotes the integral over $\bar{R}_{+}$. The operators $\tau^{a}, a \in \bar{R}_{+}$, will be called -translation operators (cf. Levitan [14]). Using these operators, Volkovich [23] obtained an analytic characterization of generalized convolutions.

Let $\mu$ be a finite measure on $\bar{R}_{+}$. We put 


$$
\left(\tau^{\mu} f\right)(x)=\int^{-} f(u) \mu^{\circ} \delta_{x}(d u)=\int^{-}\left(\tau^{a} f\right)(x) \mu(d a),
$$

where $x \in \bar{R}_{+}$and $f$ is a continuous function on $\bar{R}_{+}$.

Lemma 2.1. For every finite measure $\mu$ the operator $\tau^{\mu}$ transforms $C_{0}$ into $C_{0}$.

Proof. The assertion follows from the fact that the extended generalized convolution - is continuous in each variable separately (cf. Urbanik [21], Proposition 2.4).

Proofs of Lemmas 2.2, 2.3 and 2.4 below are similar to those for the ordinary convolution and will be omitted.

Lemma 2.2. Every $\tau^{\mu}$ is a positive bounded operator on $C_{0}$ commuting with -translation operators.

In the sequel, any operator on a function space commuting with -translation operators will be called-translation invariant.

Lemma 2.3. Let $A$ be a positive bounded -translation invariant operator on $C_{0}$. There exists a uniquely determined finite measure $\mu$ on $\bar{R}_{+}$such that

$$
A=\tau^{\mu} \text {. }
$$

Lemma 2.4. For any $\mu, \nu \in \bar{P}$

$$
\tau^{\mu} \tau^{\nu}=\tau^{\nu} \tau^{\mu}=\tau^{\mu \circ \nu}
$$

We note that

$$
\int^{-} f(u)\left(\mu^{\circ} \nu\right)(d u)=\int^{-} \int^{-}\left(\tau^{u} f\right)(v) \mu(d u) \nu(d v),
$$

where $\mu, \nu \in \bar{P}$ and $f$ is a continuous function on $\bar{R}_{+}$.

THEOREM 2.5. Let $\left\{\mu_{t}\right\}$ be an -semigroup of p.m.'s on $\bar{R}_{+}$. The formula

$$
S_{t}=\tau^{\mu_{t}} \quad(t \geq 0)
$$

defines a strongly continuous -translation invariant contraction semigroup on $C_{0}$. 
Conversely, if $\left(S_{t}\right)$ is a strongly continuous -translation invariant contraction semigroup of positive operators on $C_{0}$, then it is given by (2.7) with the same - -semigroup of p.m.'s on $\bar{R}_{+}$. The correspondence $\left\{\mu_{t}\right\} \leftrightarrow\left\{S_{t}\right\}$ is one-to-one.

Proof. From Lemmas 2.1, 2.2 and 2.4 it follows that $\left\{S_{t}\right\}$ defined by (2.7) is an -translation invariant contraction semigroup. Its strong continuity follows from Chung's remark (cf. Chung [5], p. 49). The converse statement follows from Lemma 2.3. Finally, the one-to-one correspondence $\left\{\mu_{t}\right\} \leftrightarrow\left\{S_{t}\right\}$ is a consequence of Lemma 2.2 .

Let $\left\{X_{t}\right\}$ be an -Lévy process with the transition probability given by

$$
P_{t}(x, .)=\mu_{t}^{\circ} \delta_{x} \quad\left(t \geq 0, x \in \bar{R}_{+}\right) .
$$

The corresponding semigroup $\left\{S_{t}\right\}$ can be written in the form

$$
\left(S_{t} f\right)(x)=E^{x} f\left(X_{t}\right) .
$$

By Theorem $2.5\left\{S_{t}\right\}$ is a strongly continuous semigroup on $C_{0}$, which implies that $\left\{X_{t}\right\}$ is a Feller process. Moreover, since the function $(t, x, f) \mapsto\left(S_{t} f\right)(x)$ is continuous (cf. Chung [5]), it follows that the process is a strong Markov process (cf. Blumenthal and Getoor [3], p.41). Thus we have the following theorem (cf. Chung [5], Proposition 2, p.50 and Theorem 6, p.54):

THEOREM 2.6. Every -Lévy process is a strong Markov Feller process. Consequently, it is stochastically continuous and has a version with right continuons paths having left limits.

Remark 2.7. For some generalized convolution •, there exist -Lévy processes with continuous paths. For example, the absolute value of the Brownian motion is a $*_{1,1}$-Lévy process having continuous paths.

\section{Infinitesimal generators}

The aim of this section is to study the infintesimal generators of the semigroups associated with -Lévy processes.

To begin with we introduce the following generalized differential operator:

$$
D^{\circ} f(x)=\lim _{y \rightarrow 0+} \frac{\tau^{x} f(y)-f(x)}{w(y)},
$$


where $f$ is a function in $C_{0}$ and the limit is taken in $C_{0}$-norm and the function $w($.$) is defined by$

$$
\begin{aligned}
w(y) & =1-\Omega(y), \quad 0 \leq y \leq x_{0} \\
& =1-\Omega\left(x_{0}\right), \quad y>x_{0}
\end{aligned}
$$

$x_{0}$ being a number such that $0<\Omega(y)<1$ for $0<y \leq x_{0}$. The domain of $D^{\circ}$ is denoted by $\mathfrak{D}\left(D^{\circ}\right)$.

As in Klosowska [11] and Bingham [2] we shall assume that

$$
V^{-1}=\int x^{x} \sigma_{x}(d x)<\infty,
$$

which holds true for all known examples of regular generalized convolutions.

Lemma 3.1. Let $\left\{\mu_{t}\right\}$ be an-semigroup in $(P, \circ)$. There exists a finite measure $m$ on $R_{+}$such that

$$
\frac{w(x)}{t} \mu_{t}(d x) \stackrel{w}{\rightarrow} m \quad \text { as } \quad t \rightarrow 0
$$

Proof. Since $\mu_{1}$ is $\circ$-infinitely divisible, there is a unique finite measure $m$ on $R_{+}$such that

$$
\hat{\mu}_{1}(u)=\exp \int \frac{\Omega(u x)-1}{w(x)} m(d x)
$$

by [16] Theorem 13 and [17] Theorem 1. Hence

$$
\hat{\mu}_{t}(u)=\exp \left(t \int \frac{\Omega(u x)-1}{w(x)} m(d x)\right) .
$$

Let $m_{t}(d x)=t^{-1} w(x) \mu_{t}(d x)$ for $t>0$. Then

$$
\int \frac{\Omega(u x)-1}{w(x)} m_{t}(d x)=t^{-1}\left(\hat{\mu}_{t}(u)-1\right) \rightarrow \int \frac{\Omega(u x)-1}{w(x)} m(d x) \quad(t \rightarrow 0)
$$

uniformly on every finite interval. Now the argument in the proof of [16] Theorem 13 applies and we get $m_{t} \stackrel{w}{\rightarrow} m$ as $t \rightarrow 0$.

Lemma 3.2. Suppose that (3.3) holds. Define

$$
\beta_{y}(u)=V y^{-\varkappa} u^{x} T_{y} \sigma_{x}(d u) \quad(y>0) .
$$


Then every $\beta_{y}$ is a p.m. on $R_{+}$and

$$
\beta_{y} \stackrel{w}{\rightarrow} \delta_{0} \quad(y \rightarrow 0)
$$

Proof. We have

$$
\hat{\beta}_{y}(t)=\int u^{\varkappa} \Omega(t u y) V_{\sigma_{\kappa}}(d u),
$$

which implies that $\hat{\beta}_{y}(0)=1$ and therefore $\beta_{y}$ is a p.m. Moreover, lettng $y$ tend to zero we have $\hat{\beta}_{y}(t) \rightarrow 1$. Consequently, (3.5) holds.

Let $H$ be the class of functions of the form

$$
f_{a}(x)=\exp \left(-a^{\alpha} x^{\alpha}\right) \quad\left(a>0, x \in R_{+}\right) .
$$

Lemma 3.3. Suppose that (3.3) holds. The operator $D^{\circ}$ is densely defined in $C_{0}$, and the domain $\mathfrak{D}\left(D^{\circ}\right)$ contains the class $H,(3.1)$ is equivalent to the following

$$
D^{\circ} f(x)=\lim _{y \rightarrow 0} \frac{\tau^{x} f(y)-f(x)}{V y^{x}} .
$$

Proof. When (3.3) holds, Klosowska ([11], Lemma 1) shows that

$$
\frac{w(y)}{y^{x}} \rightarrow V \quad(y \rightarrow 0),
$$

which implies that (3.1) is equivalent to (3.1'). The linear combinations of elements of $H$ are dense in $C_{0}$. Let us prove that $D^{\circ} f_{a}$ is defined for any $a>0$. By (1.2), (2.4) and (3.3) we have

$$
\begin{aligned}
& \left|\frac{\tau^{x} f_{a}(y)-f_{a}(x)}{V y^{\varkappa}}+\int \Omega(a x v) a^{\varkappa} v^{\varkappa} \sigma_{\kappa}(d v)\right|= \\
& =\left|\frac{\iint \Omega(a u v) \sigma_{\varkappa}(d v) \delta_{x} \circ \delta_{y}(d u)-\int \Omega(a x v) \sigma_{\varkappa}(d v)}{V y^{\varkappa}}+\int \Omega(a x v) a^{\varkappa} v^{\varkappa} \sigma_{\varkappa}(d v)\right| \\
& =\left|\int \Omega(a x v)\left\{\frac{\Omega(a y v)-1}{V y^{\varkappa}}+a^{\varkappa} v^{\varkappa}\right\} \sigma_{\varkappa}(d v)\right| \\
& =\left|\int \Omega\left(a x u y^{-1}\right) \frac{\Omega(a u)-1+V a^{\varkappa} u^{\varkappa}}{V y^{\varkappa}} T_{y} \sigma_{k}(d u)\right|
\end{aligned}
$$




$$
\begin{aligned}
& \leq \int\left|\Omega(a u)-1+V a^{\varkappa} u^{\varkappa}\right| V^{-1} y^{-\varkappa} T_{y} \sigma_{\varkappa}(d u) \\
& =V^{-2} \int\left|\frac{\Omega(a u)-1}{u^{\varkappa}}+V a^{\varkappa}\right| V y^{-\varkappa} u^{\varkappa} T_{y} \sigma_{\varkappa}(d u),
\end{aligned}
$$

where the integrand is a continuous bounded function of $u$ and vanishes at $u=0$. By Lemma 3.2, the last expression tends to zero as $y \rightarrow 0$, which implies that $H \subset$ $\mathfrak{D}\left(D^{\circ}\right)$ and

$$
\lim _{y \rightarrow 0} \frac{\tau^{x} f_{a}(y)-f_{a}(x)}{V y^{x}}=-\int \Omega(a x v) a^{\alpha} v^{\alpha} \sigma_{\kappa}(d v)
$$

uniformly in $x$ for every positive number $a$.

THEOREM 3.4. Suppose that (3.3) holds. Let $A$ be the infinitesimal generator of the semigroup associated with an o-Lévy process on $\bar{R}_{+}$with domain $\mathscr{D}(A)$. Then $\mathfrak{D}\left(D^{\circ}\right) \subset \mathfrak{D}(A)$ and

$$
A f(x)=\int \frac{\tau^{x} f(u)-f(x)}{w(u)} \nu(d u-\rho f(x)
$$

for $f \in \mathfrak{D}\left(D^{\circ}\right)$, where $\rho$ is a nonnegative constant and $\nu$ is a finite measure on $R_{+}$. The integrand assumes the value $D^{\circ} f(x)$ at $u=0$. The pair $(\nu, \rho)$ is uniquely determined by $A$.

Conversely, for any pair $(\nu, \rho)$, there exists a unique -Lévy process on $\bar{R}_{+}$satisfying (3.8) for all $f \in \mathfrak{D}\left(D^{\circ}\right)$.

Proof. Let $A$ be the infinitesimal generator for the semigroup $\left\{S_{t}\right\}$ given by (2.7) and (2.8). Putting

$$
\rho(t)=\mu_{t}\left(R_{+}\right) \quad(t \geq 0)
$$

and taking into account the continuity of $\left\{\mu_{t}\right\}$, we have

$$
\rho(t)=\exp (-\rho t) \quad(t \geq 0)
$$

with some $\rho \geq 0$. Let $f \in \mathfrak{D}\left(D^{\circ}\right)$. We have

$$
\begin{aligned}
A f(x) & =\lim _{t \rightarrow 0} \frac{S_{t} f(x)-f(x)}{t} \\
& =\lim _{t \rightarrow 0} \int^{-}\left[\tau^{x} f(y)-f(x)\right] \frac{1}{t} \mu_{t}(d y)
\end{aligned}
$$




$$
\begin{aligned}
& =\lim _{t \rightarrow 0}\left\{\int\left[\tau^{x} f(y)-f(x)\right] \frac{1}{w(y)} t^{-1} w(y) \mu_{t}(d y)-\frac{1-\rho(t)}{t} f(x)\right\} \\
& =\int \frac{\tau^{x} f(y)-f(x)}{w(y)} \nu(d y)-\rho f(x),
\end{aligned}
$$

where $\nu$ is the weak limit of $t^{-1} w(y) \mu_{t}(d y)$ as $t \rightarrow 0$ (Lemma 3.1), and the integrand in the last expression assumes the value $D^{\circ} f(x)$ (Lemma 3.3).

Since the last expression of the above equalities belongs to $C_{0}$ and since the convergence is boundedly pointwise, the limit can be taken in $C_{0}$-norm by the use of a general theory (Dynkin [7] Lemma 2.11). This shows that $\mathfrak{D}\left(D^{\circ}\right) \subset \mathfrak{D}(A)$ and (3.8) holds.

To prove the uniqueness of representation (3.8), use the fact $H \subset \mathfrak{D}\left(D^{\circ}\right)$ in Lemma 3.8. By (3.7) we have $D^{\circ} f_{a}(0)=-V^{-1} a^{x}$. Hence

$$
A f_{a}(0)=-V^{-1} a^{\alpha} \nu(\{0\})+\int_{(0, \infty)} \frac{\exp \left(-a^{\alpha} y^{\alpha}\right)-1}{w(y)} \nu(d y)-\rho .
$$

Since $A f_{a}(0) \rightarrow-\rho$ as $a \rightarrow 0, \rho$ is unique. Since $a^{-x}\left(A f_{a}(0)+\rho\right) \rightarrow$ $-V^{-1} \nu(\{0\})$ as $a \rightarrow \infty, \nu(\{0\})$ is unique. Moreover, if finite measures $\nu$ and $\nu^{\prime}$ satisfy

$$
\int_{(0, \infty)} \frac{\exp \left(-a^{\alpha} y^{\alpha}\right)-1}{w(y)} \nu(d y)=\int_{(0, \infty)} \frac{\exp \left(-a^{\alpha} y^{\alpha}\right)-1}{w(y)} \nu^{\prime}(d y)
$$

for all $a>0$, then $\nu=\nu^{\prime}$ on $(0, \infty)$ by the uniqueness theorem for Laplace transforms, because the above equality is written to

$$
\int_{0}^{\infty} e^{-a^{\kappa} s} d s \int_{s}^{\infty} \frac{\nu(d y)}{w(y)}=\int_{0}^{\infty} e^{-a^{\kappa} s} d s \int_{s}^{\infty} \frac{\nu^{\prime}(d y)}{w(y)}
$$

Conversely, given a pair $(\nu, \rho)$, let $\gamma$ be an -infinitely divisible p.m. on $R_{+}$ satisfying

$$
\hat{\gamma}(u)=\exp \int \frac{\Omega(u x)-1}{w(x)} \nu(d x)
$$

(cf. Urbanik [16]). Then the infinitesimal generator $A$ for the semigroup $\left\{S_{t}\right\}$ given by (2.7) with

$$
\mu_{t}(t)=\exp (-\rho t) \gamma^{o t}+(1-\exp (-\rho t)) \delta_{\infty}
$$

satisfies (3.8). It is easy to see that this $\mu_{t}$ is uniquely determined by $(\nu, \rho)$. 
A particular but very important case of o-Lévy processes is the processes induced by the characteristic measure $\sigma_{x}$.

THEOREm 3.5. Suppose that (3.3) holds. Let $A$ be the infinitesimal generator for the -Lévy process $\left\{X_{t}\right\}$ such that the $P^{0}$-distribution of $X_{1}$ is equal to $\sigma_{\varkappa}$. Then $A f=D^{\circ}$ for every $f \in \mathfrak{D}\left(D^{\circ}\right)$.

Proof. Apply Theorem 3.4. The measure $\nu$ there must satisfy

$$
\int \frac{\Omega(u x)-1}{w(x)} \nu(d x)=-u^{x}
$$

in this case by virtue of (1.2). Since the integrand assumes the value $u^{x}$ at $x=0$, we have $\nu=\delta_{0}$.

Now, by virtue of formulas $\left(3.1^{\prime}\right)$ and (3.7), we get the following examples of $D^{\circ}$ :

$\alpha$-convolutions: $\quad D^{\circ} f(x)=a^{-1} x^{1-\alpha} f^{\prime}(x)$.

Symmetric convolution: $D^{\circ} f=f^{\prime \prime}$.

Kingman convolution $*_{1, \beta}(\beta=2(s+1)>1)$ : By Gradshteyn and Ryzhik ([8], $3.381(4))$, the constant $V$ in $(3.3)$ is given by

$$
V=\frac{1}{4(s+1)} .
$$

Next, for $f \in C_{0}$ and $x, y \geq 0$ we have (cf. Urbanik [16])

$$
\tau^{x} f(y)=\frac{\Gamma(s+1)}{\sqrt{\pi} \Gamma\left(s+\frac{1}{2}\right)} \int_{-1}^{1} f\left(\left(x^{2}+2 u x y+y^{2}\right)^{\frac{1}{2}}\left(1-u^{2}\right)^{s-\frac{1}{2}} d u,\right.
$$

which together with Lemma 3.3 leads to the following formula (cf. Gradshteyn and Ryzhik [8], 3.251 (1) and 3.249 (5)):

$$
D^{\circ} f(x)=f^{\prime \prime}(x)+(2 s+1) x^{-1} f^{\prime}(x) .
$$

\section{Generalized Bernstein functions}

We say that the family $\left\{\nu_{t}\right\}$ of sub-p.m.'s on $R_{+}$is an o-semigroup if the following conditions are satisfied:

$$
\nu_{t} \circ \nu_{s}=\nu_{t+s} \quad(t, s \geq 0) .
$$


$\nu_{t} \rightarrow \delta_{0} \quad$ vaguely as $t$ tends to 0,

that is, $\int f(x) \nu_{t}(d x) \rightarrow f(0)$ as $t \rightarrow 0$ for every continuous function $f$ on $R_{+}$with compact support.

Clearly, these conditions imply that $\nu_{0}=\delta_{0}$ and $\nu_{t} \stackrel{w}{\rightarrow} \delta_{0}$ as $t \rightarrow 0$. Let $\left\{X_{t}\right\}$ be an -Lévy process on $\bar{R}_{+}$induced by an o-semigroup $\left\{\mu_{t}\right\}$ of p.m.'s (cf. $\S 2$ ). The restriction of $\left\{\mu_{t}\right\}$ to $R_{+}$, denoted by $\left\{\nu_{t}\right\}$, is an $\circ$-semigroup of sub-p.m.'s. Since every measure $\nu_{t}$ is infinitely divisible with respect to $\circ$, the characteristic function of $\nu_{t}$ is of the form (cf. Urbanik [16], [17])

$$
\hat{\nu}_{t}(u)=\exp (-t f(u)),(u, t \geq 0),
$$

where $f$ is given by

$$
f(u)=a+b u^{x}+\int(1-\Omega(u x)) m(d x),
$$

$a, b$ being nonnegative constants and $m$ being a measure on $R_{+}$vanishing at the origin such that

$$
\int w(x) m(d x)<\infty
$$

where $w($.$) is a function defined by (3.2).$

Let $F\left({ }^{\circ}\right)$ denote the set of all functions of the form (4.2). Let $S\left({ }^{\circ}\right)$ denote the set of all functions in $F\left({ }^{\circ}\right)$ corresponding to o-self-decomposable sub-p.m.'s (cf. Urbanik [17]). For the ordinary convolution the set $F\left({ }^{\circ}\right)$ coincides with the set of all Bernstein functions (cf. Berg \& Forst [1], p. 61). Hence in general case the functions in $F\left({ }^{\circ}\right)$ will be called generalized Bernstein functions, shortly - Bernstein functions.

It is evident that the set $F\left({ }^{\circ}\right)$ is a cone which does not depend upon the choice of the system of characteristic functions and is closed under the convergence that is uniform on every compact set.

Proposition 4.1. Let $\left\{\mu_{t}\right\}$ be an o-semigroup (of sub-p.m.'s) and $\left\{\nu_{t}\right\} a$ $*_{\alpha}$-semigroup $(\alpha>0)$. Then the integral

$$
\tau_{t}=\int \mu_{s} \alpha \nu_{t}(d s) \quad(t \geq 0)
$$

defines an -semigroup. 
Proof. We have, for $t, u \geq 0$,

$$
\begin{aligned}
\hat{\tau}_{t}(u) & =\int \exp \left(-s^{\alpha} f(u)\right) \nu_{t}(d s) \\
& =\exp \left(-\operatorname{tg}\left(f^{\alpha^{-1}}(u)\right)\right),
\end{aligned}
$$

$f, g$ being generalized Bernstein functions associated with $\left\{\mu_{t}\right\}$ and $\left\{\nu_{t}\right\}$, respectively.

As an immediate consequence of the above proposition we have

COROLlary 4.2. If $f \in F(\circ)$ and $g \in F\left(*_{\alpha}\right)$, then $g\left(f^{\alpha^{-1}}\right) \in F(\circ)$. In particular, if $h$ is a Bernstein function, then $h(f)$ is an-Bernstein function.

The converse statement is also true. Namely, we have

Proposition 4.3. Let $g$ be a function such that for every generalized convolution - and for every $f \in F(\circ)$ the composite function $g\left(f^{\alpha^{-1}}\right)$ belongs to $F(\circ)$. Then $g$ is $*_{\alpha}$-Bernstein function.

Proof. It follows from the fact that the function $f(x)=x^{\alpha}$ belongs to $F\left(*_{\alpha}\right)$.

Let $\circ$ and $\circ$ ' be regular generalized convolutions. Let us denote $G\left({ }^{\circ}\right)=$ $\{\hat{\mu}: \mu \in Q\}$, which is independent of the choice of the system of characteristic functions. Then we have the following inclusions:

$$
\begin{aligned}
& G\left(*_{\alpha}\right) \subset G\left({ }^{\circ}\right) \\
& F\left(*_{\alpha}\right) \subset F\left({ }^{\circ}\right) \\
& S\left(*_{\alpha}\right) \subset S\left({ }^{\circ}\right)
\end{aligned}
$$

where $0<\alpha \leq \kappa\left({ }^{\circ}\right), \kappa\left({ }^{\circ}\right)$ being the characteristic exponent of $\circ$. Moreover, Theorem 2.2 in Urbanik [18] can be formulated as follows:

THEOREM 4.4. If $G\left({ }^{\circ}\right)=G\left(\circ^{\prime}\right)$, then ${ }^{\circ}=\circ^{\prime}$.

Similarly, we have the following: 
THEOREM 4.5. The following equalities are equivalent:

(i) $\circ=\circ^{\prime}$,

(ii) $F\left({ }^{\circ}\right) \subset F\left(\circ^{\prime}\right)$,

(iii) $S\left({ }^{\circ}\right) \subset S\left({ }^{\circ}\right)$.

Proof. We shall prove that (ii) implies (i). Suppose that (ii) is true. Let $\Omega$ and $\Omega^{\prime}$ be the kernels of ${ }^{\circ}$ and ${ }^{\circ}$, respectively. By (4.2) there exist $a^{\prime}, b^{\prime}$ and $m^{\prime}$ such that

$$
1-\Omega(u)=a^{\prime}+b^{\prime} u^{\varkappa\left(0^{\prime}\right)}+\int\left(1-\Omega^{\prime}(u x)\right) m^{\prime}(d x) .
$$

Since $\Omega(0)=1$ and $\Omega(u)$ is bounded, we have $a^{\prime}=b^{\prime}=0$. Similarly, there is a measure $m$ such that

$$
1-\Omega^{\prime}(u)=\int(1-\Omega(u y)) m(d y)
$$

Hence

$$
\begin{aligned}
1-\Omega(u)=\iint(1- & \Omega(u x y)) m^{\prime}(d x) m(d y) \\
& =\int(1-\Omega(u x)) H(d x),
\end{aligned}
$$

where

$$
H(d x)=\int m^{\prime}(d x / y) m(d y)
$$

In particular, we have the equation

$$
\begin{aligned}
\int_{0}^{x_{0}}(1-\Omega(x)) H(d x) & =\int_{0}^{x_{0}} w(x) H(d x) \\
& \leq 1-\Omega(1),
\end{aligned}
$$

where $x_{0}$ is the same as in (3.2). On the other hand, by formula (41) in Urbanik [16] and by Fatou's lemma

$$
1 \geq \liminf _{t \rightarrow 0} \int \frac{1-\Omega(t x)}{1-\Omega(t)} H(d x) \geq \int x^{\kappa(\circ)} H(d x) .
$$

Consequently, $H$ is finite on every half-liine $[A, \infty)(A>0)$, which together with (4.6) implies that $H$ satisfies the condition (4.3). Therefore, by (4.5) and by 
uniqueness of the representation (4.2), it follows that $H=\delta_{1}$ and consequently, $m^{\prime}=b \delta_{c}$ for some positive $b, c$, which implies that

$$
\Omega(u)=b \Omega^{\prime}(c u)+1-b, \quad(u \geq 0) .
$$

Let $p$ be a positive number less than $\min \left(\kappa(\circ), \kappa\left(\circ^{\prime}\right)\right)$. Let $\sigma_{p}$ and $\sigma_{p}^{\prime}$ be $\circ$-stable and $\circ^{\prime}$-stable measures, respectively, with the same exponent $p$ (cf. Urbanik [16]). Integrating both sides of (4.7) with respect to $\sigma_{p}$ and $\sigma_{p}^{\prime}$ and using Fubini's theorem, we get the equation

$$
\int \exp \left(-y^{p} u^{p}\right) \sigma_{p}^{\prime}(d y)=b \int \exp \left(-c^{p} x^{p} u^{p}\right) \sigma_{p}(d x)+1-b
$$

Notice that $\sigma_{p}$ and $\sigma_{p}^{\prime}$ do not have point mass at 0 (cf. Urbanik [19] Lemma 2.2; the proof becomes simpler since our generalized convolutions are regular).

Letting $t \rightarrow 0$ in the last equation, we get $b=1$ and $\Omega(u)=\Omega^{\prime}(c u)(u \geq 0)$. Consequently, ${ }^{\circ}=0^{\prime}$ which completes the proof that (ii) implies (i). The proof that (iii) implies (i) is similar and is omitted.

As a consequence of the above theorem we have the following characterization of $\alpha$-convolutions:

ThEorem 4.6. Let $0<\alpha \leq \kappa\left({ }^{\circ}\right)$. Then the equality ${ }^{\circ}=*_{\alpha}$ (and necessarily $\alpha=\kappa(\circ))$ holds if and only if, for any $\circ^{\prime}, g \in F\left(\circ^{\circ}\right)$, and $f \in F\left(\circ^{\prime}\right)$, the composite function $g\left(f^{1 / \alpha}\right)$ belongs to $F\left(\circ^{\prime}\right)$.

Proof. The "only if" part follows from Corollary 4.2. To prove the "if" part let us take $g$ from $F\left({ }^{\circ}\right), \circ^{\prime}=*_{\alpha}$, and $f(x)=x^{\alpha}$. By the assumption the composite function $g\left(f^{1 / \alpha}\right)=g$ belongs to $F\left(*_{\alpha}\right)$, which implies $F(\circ) \subset F\left(*_{\alpha}\right)$ and, by Theorem $4.5,{ }^{\circ}=*_{\alpha}$.

We conclude this section by giving a sufficient condition for transience of -Lévy processes.

THEOREM 4.7. Suppose that the kernel $\Omega$ is nonnegative. Then every non-constant - Lévy process on $R_{+}$is transient.

Proof. Let $\mu_{t}$ and $f$ be the $\circ$-semigroup and the $\circ$-Bernstein function associated with a non-constant -Lévy process $\left\{X_{t}\right\}$. Thus $f$ is not identically zero. By Lemma 2.1 in Urbanik [20] the set of zeros of $f$ has Lebesgue measure zero. 
Further, for every continuous nonnegative function $g$ on $R_{+}$with compact support there exist positive constants $a$ and $b$ such $f(b)>0$ and for every $u \geq 0$

$$
g(u) \leq a \Omega(b u)
$$

which implies that

$$
\begin{aligned}
\int E^{x} g\left(X_{t}\right) d t & \leq a \int E^{x} \Omega\left(b X_{t}\right) d t \\
& =a \iint \Omega(b u)\left(\delta_{x} \circ \mu_{t}\right)(d u) d t \\
& =a \Omega(b x) \int \exp (-t f(b)) d t \\
& =a / f(b)<\infty .
\end{aligned}
$$

Remark 4.8. For some generalized convolution $\circ$, there exist non-constant recurrent -Lévy processes. In such a case the kernel $\Omega$ must take negative values somewhere (see Kingman [10], Theorem 10, for a transience criterion for $*_{1, \beta}$-Lévy processes).

\section{Self-similar -i.i. processes}

This section continuous the line of research of Lamperti [13] and Sato [15].

Consider an - -i.i. process $\left\{X_{t}\right\}$ on $\bar{R}_{+}$with transition probability $P_{s, t}$ given by (2.2). We say that the process $\left\{X_{t}\right\}$ is $H$-self-similar $(H>0)$, if it is $H$-self-similar as a Markov process, namely, if for any $a>0$ and $x \in \bar{R}_{+}$the finite-dimensional $P^{x}$-distributions of $\left\{X_{t}\right\}$ are identical with the finitedimensional $P^{a^{H} x}$-distribution of $\left\{a^{-H} X_{a t}\right\}$.

The following theorems stand for analogues of Sato's results [15]:

THEOREM 5.1. If $\left\{X_{t}\right\}$ is an $H$-self-similar -i.i. process, then for every $t$ the $P^{0}$-distribution of $X_{t}$ is o-decomposable.

THEOREM 5.2. Suppose that $\mu$ is an o-self-decomposable measure in $\bar{P}$ and $\mu \neq$ $\delta_{\infty}$. Then for any $H>0$ and $t_{0}>0$ there exists a unique $H$-self-similar-i.i. process $\left\{X_{t}\right\}$ such that $\mu$ is the $P^{0}$-distribution of $X_{t_{0}}$. The uniqueness here is in the sense of finite-dimensional distributions.

A natural question arises: What can be said about the $P^{x}$-distribution of $X_{t}$ 
for $x>0$ ? And, more generally, what can be said about the $P^{\nu}$-distribution of $X_{t}$ for $\nu \in \bar{P}$ ? The following theorem answers these questions and gives a characterization of $\alpha$-convolutions by self-similarity.

THEOREM 5.3. Let $\left\{X_{t}\right\}$ be $H$-self-similar -i.i. process such that $\mu_{0, t} \neq \delta_{\infty}$ for every $t>0$. Let $\nu \in \bar{P}$. Then, the $P^{\nu}$-distribution of $X_{t}$ is - -self-decomposable for $e v$ ery $t>0$, if and only if $\nu$ is -self-decomposable.

Consequently, the following two statements are equivalent:

(i) There exists an $H$-self-similar o-i.i. process $\left\{X_{t}\right\}$ and a point $x(0<x$ $<\infty)$ such that $\mu_{0, t} \neq \delta_{\infty}$ for every $t>0$ and the $P^{x}$-distribution of $X_{t}$ is - self-decomposable for every $t>0$.

(ii) 。 is an $\alpha$-convolution for some $\alpha(0<\alpha<\infty)$.

A p.m. $\mu \in \bar{P}$ is said to be $\circ$-stable if, for any pair $a, b$ in $(0, \infty)$, there exists $c \in(0, \infty)$ such that $T_{a} \mu \circ T_{b} \mu=T_{c} \mu$. If $\mu \in \bar{P}$ is o-stable, then $\mu=\delta_{\infty}$ or $\mu \in P$.

THEOREM 5.4. Let $\left\{X_{t}\right\}$ be a non-constant -Lévy process. Then it is self-similar if and only if the $P^{0}$-distribution of $X_{1}$ is o-stable. If the stable index is $\alpha$, then the order $H$ of self-similarity is $\alpha^{-1}$.

Proof of Theorem 5.1. Note that for any $t>0$ and $x \in \bar{R}_{+}$the $P^{x}$-distribution of $X_{t}$ is equal to $\mu_{0, t}{ }^{\circ} \delta_{x}$. Hence and by $H$-self-similarity of the process we have, for every $c=\frac{s}{t}>1$ and $a=c^{-H}$,

$$
\begin{aligned}
\mu_{0, t} & =\text { the } P^{0} \text {-distribution of } c^{-H} X_{c t} \\
& =T_{a} \mu_{0, s}=T_{a} \mu_{0, t}{ }^{\circ} T_{a} \mu_{t, s},
\end{aligned}
$$

which proves that the $P^{0}$-distribution of $X_{t}$ is o-self-decomposable.

Proof of Theorem 5.2. Suppose that $\mu$ is o-self-decomposable in $\bar{P}$. Then for any $0 \leq s<t$ there exist a unique p.m. $\mu_{s, t}$ from $\bar{P}$ such that

$$
T_{t} \mu=T_{s} \mu^{\circ} \mu_{s, t}
$$

which implies the following equality

$$
T_{c} \mu_{s, t}=\mu_{c s, c t, \quad(0 \leq s<t, c<0) .}
$$

Then the family $\left\{\mu_{s, t}\right\}$ satisfies (2.1) and induces an o-i.i. process $\left\{Y_{t}\right\}$ with tran- 
sition probability (2.2). We claim that the process is 1 -self-similar.

Denote the indicator function of a set $B$ by $1_{B}$. Given $x \in \bar{R}_{+}, a>0,0 \leq t_{1}$ $<\cdots<t_{n}$ and $B=B_{1} \times \cdots \times B_{n}, B_{j}$ 's being Borel subsets of $\bar{R}_{+}$, we have by virtue of (2.2) and (5.1),

$$
\begin{aligned}
& P^{x}\left(Y_{t_{1}} \in B_{1}, \ldots, Y_{t_{n}} \in B_{n}\right)= \\
& =\int^{-} P_{0, t_{1}}\left(x, d x_{1}\right) \cdots \int^{-} P_{t_{n-1}, t_{n}}\left(x_{n-1}, d x_{n}\right) 1_{B}\left(x_{1}, \ldots, x_{n}\right) \\
& =\int^{-} \mu_{0, t_{1}} \circ \delta_{x}\left(d x_{1}\right) \cdots \int^{-} \mu_{t_{n-1}, t_{n}} \circ \delta_{x_{n-1}}\left(d x_{n}\right) 1_{B}\left(x_{1}, \ldots, x_{n}\right) \\
& =\int^{-} T_{a}\left[\mu_{0, t_{1}}{ }^{\circ} \delta_{x}\right]\left(a d x_{1}\right) \cdots \int^{-} T_{a}\left[\mu_{t_{n-1}, t_{n}}{ }^{\circ} \delta_{x_{n-1}}\right]\left(a d x_{n}\right) 1_{B}\left(x_{1}, \ldots, x_{n}\right) \\
& =\int^{-} \mu_{0, a t_{1}}{ }^{\circ} \delta_{a x}\left(a d x_{1}\right) \cdots \int^{-} \mu_{a t_{n-1}, a t_{n}}^{\circ} \delta_{a x_{n-1}}\left(a d x_{n}\right) 1_{B}\left(x_{1}, \ldots, x_{n}\right) \\
& =\int^{-} \mu_{0, a t_{1}}{ }^{\circ} \delta_{a x}\left(a d x_{1}\right) \cdots \int^{-} \mu_{a t_{n-1}, a t_{n}}{ }^{\circ} \delta_{a x_{n-1}}\left(d x_{n}\right) 1_{B}\left(a^{-1} x_{1}, \ldots, a^{-1} x_{n}\right) \\
& =P^{a x}\left(a^{-1} Y_{a t_{1}} \in B_{1}, \ldots, a^{-1} Y_{a t_{n}} \in B_{n}\right) .
\end{aligned}
$$

This shows that $\left\{Y_{t}\right\}$ is a 1 -self-similar Markov process. Moreover, we have $\mu=$ $\mu_{0,1}$ and, therefore, $\mu$ is the $P^{0}$-distribution of $Y_{1}$.

Now let $H$ and $t_{0}$ be arbitrary positive numbers. Putting $X_{t}=Y_{t_{0}^{-H} t^{H}}$ we get a required process.

The uniqueness of $\left\{X_{t}\right\}$ follows from the fact that the transition probability $P_{s, t}$ is uniquely determined by $\mu$. Namely, for any $s<t$ and $x \in \bar{R}_{+}$we have

$$
T_{\left(t_{0} / t\right)^{-H}} \mu^{\circ} \delta_{x}=T_{\left(t_{0} / t\right)^{-H}} \mu \circ P_{s, t}(x, .) .
$$

Proof of Theorem 5.3. Suppose that $\left\{X_{t}\right\}$ is an $H$-self-similar o-i.i. process such that $\mu_{0, t} \neq \delta_{\infty}$ for every $t>0$. By Theorem 5.1 the $P^{0}$-distribution $\mu_{0, t}$ of $X_{t}$ is o-self-decomposable for every $t \geq 0$. If $\nu \in \bar{P}$, then the $P^{\nu}$-distribution of $X_{t}$ equals $\nu \circ \mu_{0, t}$. Let $\mu_{0,1}\left(R_{+}\right)=a$. Then $\mu_{0, t}\left(R_{+}\right)=a$ for every $t>0$, since $\mu_{0, t}=$ $T_{t^{H}} \mu_{0,1}$. We have $\mu_{0, t} \rightarrow a \delta_{0}+(1-a) \delta_{\infty}$ as $t \rightarrow 0$. Hence $\nu \circ \mu_{0, t}$ is $\circ$-selfdecomposable for every $t>0$ if and only if $\nu$ is - -self-decomposable. In particular, if there exists a point $x(0<x<\infty)$ such that the $P^{x}$-distribution of $X_{t}$ is --self-decomposable for every $t>0$, then the p.m. $\delta_{x}$ must be decomposable in the sense that there exist p.m.'s $\tau_{1}, \tau_{2}$ other than $\delta_{0}$ such that $\delta_{x}=\tau_{1}{ }^{\circ} \tau_{2}$, and hence the generalized convolution o is an $\alpha$-convolution $(0<\alpha<\infty)$ by a theorem of Kucharczak [12]. Conversely, if $\circ$ is an $\alpha$-convolution and the process is $H$-self-similar and o-i.i., then, for every $x \in \bar{R}_{+}$, the p.m. $\delta_{x}$ is o-self- 
decomposable and the $P^{x}$-distribution of $X_{t}(t>0)$ is o-self-decomposable.

Proof of Theorem 5.4. Suppose that $\left\{X_{t}\right\}$ is a non-constant o-Lévy process induced by an $\circ$-semigroup $\left\{\mu_{t}\right\}$. Then $\mu_{t} \neq \delta_{\infty}$ for every $t>0$. If the process is $H$-self-similar, then $\mu_{t}=T_{t^{H}} \mu_{1}$ and $\mu_{t}\left(R_{+}\right)=1$ for every $t>0$, and hence $\mu_{1}$ is - -stable of index $H^{-1}$. Conversely, if $\mu_{1}$ is o-stable of index $\alpha$, then the process is $\alpha^{-1}$-self-similar, which is proved by argument similar to the proof of Theorem 5.1 .

Acknowledgement. The author would like to express his sincere gratitude to Professors K. Sato, M. Yamazato and M. Breger for every helpful discussions during his stay in Nagoya and West Berlin. He is deeply thankful to the referee for important suggestions and corrections in the paper (Proof of the inclusion $\mathfrak{D}\left(D^{\circ}\right) \subset \mathfrak{D}(A)$ in Section 3, Proof of Lemma 3, constants in the examples of Kingman convolution and $n$-symmetric convolutions,...) and for many valuable insertions given in the referee's report on the paper.

\section{REFERENCES}

[1] Berg, C., and Forst, G. Potential theory on locally compact Abelian groups, Springer-Verlag, Berlin Heidelberg New York (1975).

[2] Bingham, N.H. On a theorem of Klosowska about generalized convolutions, Coll. Math., 48, (1984), 117-125.

[3] Blumenthal, R. M., Getoor, R. K. Markov processes and potential theory, Academic Press, New York, (1968).

[4] Cambanis, S., Keener, R. and Simons, G. On $\alpha$-symmetric multivariate distributions, J. Multivariate Anal., 13, (1983), 213-233.

[5] Chung, K.L. Lectures from Markov processes to Brownian motion, SpringerVerlag, New York Heidelberg Berlin, (1982).

[6] Dharmadhikari, S.W. and Joagdev, K. Unimodality, convexity and applications, Academic Press, San Diego (1988).

[7] Dynkin, E. B. Markov Processes, I. Springer-Verlag, Berlin (1965).

[8] Gradshteyn, I.S. and Ryzhik, I.M. Tables of integrals, series and products, Academic Press, San Diego (1980).

[9] Hudson, W.N., Mason, J. David, Operator self-similar processes in a finitedimensional space, Trans. Amer. Math. Soc., 273 (1) (1982), 281-297.

[10] Kingman, J.F.C. Random walks with spherical symmetry, Acta Math., 109 (1963), $11-53$.

[11] Klosowska, M. On the domain of attraction for generalized convolution algebras. Re. Roumaine Math. Pures Appl. 22 (1977), 669-677.

[12] Kucharczak, J., Decomposability of point measures in generalized convolution algebras, Colloq. Math., 55 (1988). 163-167.

[13] Lamperti J., Semistable stochastic processes, Trans. Amer. Math. Soc., 104 (1962), 
62-78.

[14] Levitan B.M., Generalized translation operators and some of their applications, Israel Program for Scientific Translations, Jerusalem 1962.

[15] Sato, K., Distributions of class $L$ and self-similar processes with independent increments, in "White Noise Analysis" edited by T. Hida et al., World Scientific, Singapore, (1990) pp. 360-373.

[16] Urbanik, K. Generalized convolutions, Studia Math., 23 (1964), 217-245.

[17] - Generalized convolutions II, Studia Math., 45 (1973), 57-70.

[18] — Generalized convolutions III, Studia Math., 80 (1984), 167-189.

[19] - Generalized convolutions IV, Studia Math., 83 (1986), 57-95.

[20] - Domain of attraction and moments, Probab. Math. Statist., 8 (1987), 89-101.

[21] - Quasi-regular generalized convolutions, Colloq. Math., 55 (1988), 147-162.

[22] - Generalized convolutions V, Studia Math., 91 (1988), 153-178.

[23] Vol'kovich, V.E., On an analytical description of Urbanik algebras, Izv. Akad. Nauk. UzSSR Ser. Fiz-Math. Nauk, 5 (1979), 12-17.

[24] Wintner A., Asymptotic distributions and infinite convolutions, Edwards Brothers, Ann Arbor, Michigan (1938).

Institute of Mathematics, Hanoi

P.O. Box 631 Boho, 10000 Hanoi, Vietnam 